Errata a:

Calcular e apresentar tamanhos do efeito em trabalhos científicos (1): As limitações do $p<0,05$ na análise de diferenças de médias de dois grupos

Erratum to:

Calculating and reporting effect sizes on scientific papers (1): $p<0.05$ limitations in the analysis of mean differences of two groups

Revista Portuguesa de Investigação Comportamental e Social

Portuguese Journal of Behavioral and Social Research

Fevereiro, Volume 1, Edição 1 (2015)

February, Volume 1, Issue 1 (2015)

Helena Espírito-Santo, PhD (1), Fernanda Daniel, PhD (1)

(1) Instituto Superior Miguel Torga, Coimbra, Portugal.

Errata [Erratum]

Citar este artigo como [Cite this article as]:

Espírito-Santo, H., Daniel, F. (2015). Calcular e apresentar tamanhos do efeito em trabalhos científicos (1): As limitações do $p<0,05$ na análise de diferenças de médias de dois grupos [Calculating and reporting effect sizes on scientific papers (1): $p<0.05$ limitations in the analysis of mean differences of two groups]. Revista Portuguesa de Investigação Comportamental e Social, 1(1), 3-16. doi: 10.7342/ismt.rpics.2015.1.1.14

A versão online do artigo original pode ser encontrada no seguinte doi: [The online version of the original article can be found under doi]: http://dx.doi.org/10.7342/ismt.rpics.2015.1.1.14

\title{
Errata a [Erratum to]:
}

Revista Portuguesa de Investigação Comportamental e Social [Portuguese Journal of Behavioral and Social Research], 1(1), 3-16. doi:10.7342/ismt.rpics.2015.1.1.14

Os erros seguintes requerem correção no artigo:

[The following errors require correction in the article:]

\section{1) Página $9,2^{\text {a }}$ coluna, $3^{\circ}$ parágrafo [Page 9 , 2nd column, 3rd paragraph]}

"Esta percentagem associa-se à probabilidade no extremo superior do valor de $Z$ da equação seguinte:

$$
Z=\frac{\left|M_{1}+M_{2}\right|}{\sqrt{\frac{D P_{1}^{2}+D P_{2}^{2}}{2}}}
$$

Devia ter aparecido:

[Should have appeared as:]

"Esta percentagem associa-se à probabilidade no extremo superior do valor de $Z$ das equações seguintes:

$$
d=\frac{\left|M_{1}-M_{2}\right|}{\sqrt{\frac{D P_{1}^{2}+D P_{2}^{2}}{2}}} \text { e } Z=\frac{d}{\sqrt{2}}
$$


2) Folha de Cálculo, panilha "Amostras Independentes", caixa das estimativas e interpretações [Spreadsheet, "Independent Samples" sheet, box of estimates and interpretations]

Interpretação (fórmula no campo)

$=\mathrm{SE}(\mathrm{D} 20<=0,19 ; "$ Efeito insignificante"; $\mathrm{SE}(\mathrm{E}(\mathrm{D} 20>=0,199 ; \mathrm{D} 20<0,5) ; "$ Efeito

pequeno"; $\mathrm{SE}(\mathrm{E}(\mathrm{D} 20>=0,499 ; \mathrm{D} 20<=0,79) ; " E f e i t o$ médio";SE(E(D20>=0,799;D20<=1,29);"Efeito grande"; $S E(D 20>=1,299 ; " E f e i t o$ muito grande")))))

Devia ter aparecido:

[Should have appeared as:]

=SE(B20:E28 <= 0 + B20:E28,19; "Efeito insignificante"; $\mathrm{SE}(\mathrm{E}(\mathrm{D} 20>=0,199$ + D20 < 0,5);"Efeito pequeno"; SE(E(D20 >=

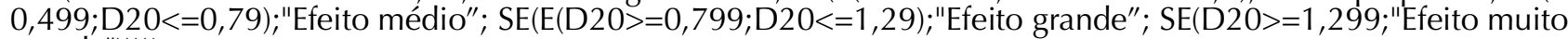
grande")))))

As autoras lamentam os erros.

[The authors regret the errors.] 